\title{
Multi-way Array Decomposition on Acoustic Source Separation for Fault Diagnosis of a Motor-Pump System
}

\author{
Anindita Adikaputri Vinaya ${ }^{1}$, Dhany Arifianto ${ }^{2}$ \\ ${ }^{1}$ Department of Engineering Management, Universitas Internasional Semen Indonesia, Indonesia \\ ${ }^{2}$ Department of Engineering Physics, Institut Teknologi Sepuluh Nopember, Indonesia
}

\begin{tabular}{l} 
Article Info \\
\hline Article history: \\
Received Sep 1, 2017 \\
Revised Nov 18, 2017 \\
Accepted Nov 30, 2017 \\
\hline
\end{tabular}

Keyword:

Acoustic emission

Fault diagnosis

Motor-pump system

Multi-way array

\begin{abstract}
In this study, we propose a multi-way array decomposition approach to solve the complexity of approximate joint diagonalization process for fault diagnosis of a motor-pump system. Sources used in this study came from drive end-motor, nondrive end-motor, drive end pump, and nondrive end pump. An approximate joint diagonalization is a common approach to resolving an underdetermined cases in blind source separation. However, it has quite heavy computation and requires more complexity. In this study, we use an acoustic emission to detect faults based on multi-way array decomposition approach. Based on the obtained results, the difference types of machinery fault such as misalignment and outer bearing fault can be detected by vibration spectrum and estimated acoustic spectrum. The performance of proposed method is evaluated using MSE and LSD. Based on the results of the separation, the estimated signal of the nondrive end pump is the closest to the baseline signal compared to other signals with LSD is 1.914 and MSE is 0.0707 . The instantaneous frequency of the estimated source signal will also be compared with the vibration signal in frequency spectrum to test the effectiveness of the proposed method.
\end{abstract}

Copyright (c) 2017 Institute of Advanced Engineering and Science. All rights reserved.

Corresponding Author:

Anindita Adikaputri Vinaya,

Department of Engineering Management,

Universitas Internasional Semen Indonesia,

Veteran Street, Gresik, East Java 61121, Indonesia.

Email: anindita.vinaya@uisi.ac.id

\section{INTRODUCTION}

Predictive maintenance plays an important role in the industry. Recently, research related to engine fault detection based on acoustic emission is being developed to increase the level of security and safety for operators in the industry. Research related to fault detection has been developed since 1969 by assuming no noise from other machines that may affect the diagnostic result. Diagnostic of machinery condition can be done by observing the location of the instantaneous frequency of the acoustic signal results [1]. Based on the previous research by Lebaroud, the instantaneous frequency can be used to identify the rotor and stator faults [2]. The acoustic signal can be recognized by Fourier transform [1], wavelet transform [3], neural network [4] and etc.

In 1988, blind source separation methods have been widely used to solve signal separation problems by using independent component analysis approach. Blind source separation is a mixed signal problemsolving approach in which the only known information is the mixture. There is no information regarding the source signal and the mixing process [5]. This method was successfully used for solving signal separation problems in the determined case (the number of sensors equal to the number of sources) using independent component analysis (ICA) [6], the overdetermined case (the number of sensors more than the number of sensors) and the underdetermined case (the number of sensors less than the number of sources) using approximate joint diagonalization (AJD) approach [5], [7]. 
Signal separation in the underdetermined case is a challenging problem to be resolved due to the lack of information that can be used to estimate the source machine. Blind source separation with AJD method widely used to separate speech signal, music signal and an acoustic signal from a machine. The approach is used to obtain the mixing matrix. In 2015 Cui et al. tried to diagnose the failure of the bearings based on vibration signals using a method based approach null-space pursuit (NSP) to solve underdetermined problem. NSP is used to estimate the differential operator from vibration model of bearing fault. In that study, the approach used to estimate the mixing matrix is AJD [8]. In previous research, [9] we have succeeded in separating the mixed signal so it can be used to determine the condition of the engine by using AJD approach [9].

If the mixing process is stationary, then the mixing matrix can be obtained by AJD. The determining process of the mixing matrix using AJD is quite complex. A non-square matrix has to be converted into a square matrix that requires estimation of a demixing matrix, unitary matrix, and selection autoterm or crossterm criterion. The method involves quite heavy computation [8], [10]. In order to reduce this complexity, the AJD approach at each bin frequency can be approximated by a multi-way array decomposition approach. The approach used by Nion et al. in 2010 [10]. Based on his research, the multiway array decomposition has a fast computation and good performance to solve the problems of speech signal separation in an underdetermined case with crosstalk reduction techniques. In 2015 , the multi-way array decomposition method is also used by Müller et al. to detect faults in HVAC system using qualitative model approach. Based on the result obtained, multi-way array decomposition can reduce the complexity of model derivation [11]. The results showed the effectiveness of the proposed approach.

In this study, we propose a multi-way array decomposition method to simplify the separation process for fault diagnosis. We conduct an experiment using acoustic emissions from a motor-pump system in the real plant. The recording of acoustic signals from the machine will be done in 2 stages that are the recording of the baseline and mixed signals. The baseline signals will be used as reference the original signals from the sources. In order to test the accuracy and effectiveness of the proposed approach, sound signal separation results will be compared with data taken with the vibration sensor.

\section{SYSTEM OVERVIEW}

\subsection{Blind Source Separation}

Blind source separation is a common method used to separate mixed signals. In order to separate the mixed signals and estimate the signal source, signal mixing process modeled previously. The mixing process configuration with two sensors and two sources (determined case) can simply be expressed as:

$$
\left[\begin{array}{l}
C_{1} \\
C_{2}
\end{array}\right]=\left[\begin{array}{ll}
A_{11} & A_{12} \\
A_{21} & A_{22}
\end{array}\right] \cdot\left[\begin{array}{l}
B_{1} \\
B_{2}
\end{array}\right]
$$

where $\mathrm{A}$ is the mixing matrix, $\mathrm{B}$ is the signal source, and $\mathrm{C}$ is a mixed signal. By performing the inverse of a matrix A, B will be obtained which is the source of the signal in the frequency domain. Therefore, the mixing matrix A must be obtained previously. The separation process can be directed to the finite impulse response to simplify the process.

$$
V(t)=\sum_{d=0}^{D-1} W(i) \cdot C(t-d)
$$

where $\mathrm{V}(\mathrm{t})=\left[\mathrm{v}_{1}(\mathrm{t}), \mathrm{v}_{2}(\mathrm{t}), \ldots . \mathrm{v}_{\mathrm{n}}(\mathrm{t})\right]^{\mathrm{T}}$ is an independent estimation of each source, $\mathrm{D}$ is the maximum channel length, and $\mathrm{W}$ is the demixing matrix. In order to estimate $\mathrm{v}(\mathrm{t})$ can be done by using the Kullback-Leibler divergence as a criterion for independency [5]. If the matrix A can not be inverted, it is necessary to use AJD with assumed independent sources. In order to simplify the estimating process of mixing matrix, multi-way array decomposition model can be developed [10], [11].

\subsection{Multi-way Array Decomposition}

In multi-way array decomposition, the mixed signal will be divided into several sub-blocks which each sub-block comprised of samples in a few blocks. Signal separation method with multi-way array decomposition begins by changing the signal from the time domain to the frequency domain after windowing process and frame blocking. Furthermore, the signal $C(f)$ will be used to search for autocorrelation matrix $R_{c}$ by calculating the cross-correlation of each point in the field of time-frequency. Matrix $R_{c}$ is sized mxmxPxsT where $\mathrm{P}$ is the time blocks along the frequency bin, sT is the length of STFT, and $\mathrm{m}$ is the number of sensors. By using multi-way array decomposition on $\mathrm{R}_{\mathrm{c}}$ at any frequency, we can get the mixing matrix A and demixing matrix $\mathrm{W}[11]$. 


$$
\begin{aligned}
& R_{c}(f)=\left[A(f) \odot A^{*}(f)\right] \cdot B^{T}(f) \\
& R_{c}(f, p)=A(f, p) \cdot R_{b}(f, p) \cdot A^{H}(f)
\end{aligned}
$$

where $R_{c}(f, p)=E\left[c(f, p) c^{H}(f, p)\right]$ and $R_{b}(f, p)=E\left[b(f, p) b^{H}(f, p)\right]$. Each element of the $\mathrm{R}_{\mathrm{c}}(\mathrm{f})$ is $r_{m 1 m 2 p}^{c}(f)$, each element of $A(f)$ is $a_{m, n}(f)$ and each element of the diagonal of $R_{b}(f, p)$ is $v_{p, n}(f)$ so that the equation may be written as follows:

$$
r_{m 1 m 2 p}^{c}(f)=\sum_{n=0}^{N} a_{i j}(f) \cdot v_{p, n}(f) \cdot a_{m 2, n}^{*}(f)
$$

Multi-way array $R_{c}$ (f) is constructed from the matrix elements in the Equation (5) where $n$ is the numbers of sources. The equation is a conjugate symmetric of parallel factor decomposition used to get the mixing matrix $A(f)$ and the matrix of power spectra $V(f)$ through the permutation process. The mixing matrix $A(f)$ and the source power spectra $V(f)$ can be estimated with alternating least squares algorithm by minimizing the Frobenius norm in Equation (6).

$$
R_{c}(f)=E(f) \cdot \Sigma(f) \cdot G^{H}(f)
$$

In the structure of the Khatri-Rao, $\mathrm{E}(\mathrm{f}), \Sigma(\mathrm{f})$, and $\mathrm{G}^{\mathrm{H}}(\mathrm{f})$ is exemplified matrix $\mathrm{I}, \mathrm{J}$, and $\mathrm{K}$ which is a constituent mixed matrix C. For matrices $I$, J, and $K$, the mixed matrix $C$ operated with the function $Z_{i}, Z_{j}$, and $Z_{k}$. The algorithm begins with determining the profile matrix $I$ by matrix $K \odot J=Z_{i}$ so that $I=C \cdot Z_{i}^{+}$. In order to determine the profiles matrix $\mathrm{J}$ then $\mathrm{C}=\mathrm{J} .(\mathrm{K} \odot \mathrm{I})^{\mathrm{T}}=\mathrm{J} \cdot \mathrm{Z}_{\mathrm{j}}^{\mathrm{T}}$, so $\mathrm{J}=\mathrm{C} . \mathrm{Z}_{\mathrm{j}}^{+}$and to determine the profile matrix $\mathrm{K}$ then $\mathrm{C}=\mathrm{K} .(\mathrm{J} \odot \mathrm{I})^{\mathrm{T}}=\mathrm{K} \cdot \mathrm{Z}_{\mathrm{k}}^{\mathrm{T}}$, so $\mathrm{K}=\mathrm{C} \cdot \mathrm{Z}_{\mathrm{k}}{ }^{+}$. Profile matrices $\mathrm{I}$, J, and $\mathrm{K}$ will be iterated until it reaches a minimum fitting function [10], [11].

$$
\begin{aligned}
& R s S=\sum_{1=1}^{I} \sum_{j=1}^{J} \sum_{k=1}^{K}\left(c_{i j k}-\hat{c}_{i j k}\right)^{2} \\
& \% f i t=\left(1-\frac{R s s}{\sum_{1=1}^{I} \Sigma_{j=1}^{J} \Sigma_{k=1}^{K} c_{i j k}{ }^{2}}\right)
\end{aligned}
$$

\subsection{Fault Identification}

Machinery faults will disrupt the production process in the industry. It is caused, due to several factors such as the effects of temperature, lubrication, design and installation, and etc. In order to identify faults, the pattern of the vibration spectrum must be analyzed. Each machine has different patterns, depending on the level and type of defect in the machine. Misalignment and bearing fault are examples of the fault that can occur in rotating machinery. Misalignment is a condition when the center line of the geometry of the two coupled machines is non-linear along the axis line of the machine during operation. It refers to the mounting condition. On the other hand, rolling element bearing faults can be caused by $40 \%$ of improper lubrication factor, $30 \%$ from improper mounting and $10 \%$ of the lifetime [12].

Misalignment can be identified by a high amplitude $1 \mathrm{x}$ and $2 \mathrm{x}$ in the frequency domain. In a severe case, a high and strong peak $3 \mathrm{x}$ or $4 \mathrm{x}$ is still categorized as misalignment. Identification of bearing fault also varies depending on the types and levels of the defect. Bearings have static and dynamic components. Ball bearings include dynamic components that are specialized to bear a high work. The ball bearing is between the inner race and the outer race. It moves inside the cage. When there is friction between the ball bearings with other components then the resulting frequency will be different from before. The types of defect depend on the interaction between the roller element bearing with other parts such as the outer race, inner race, and etc. In a severe case, bearing fault is marked with high peaks in the upper frequencies that are not a harmonics [13].

\section{SEPARATION PROCESS AND EXPERIMENT}

\subsection{Separation Process}

In this study, an acoustic signal in the time domain as shown in Figure 1 will be transformed in time-frequency domain using short-time Fourier transform. Previously, the signal must be formed into chunks of limited time through the sampling process. Furthermore, a signal sampling results will be formed into a number of frames in which a frame is made up of several samples (frame blocking). It will be merged back through windowing process. The process is useful for reducing the effects of discontinuities in the pieces of the signal before Fourier transformation process [5]. 


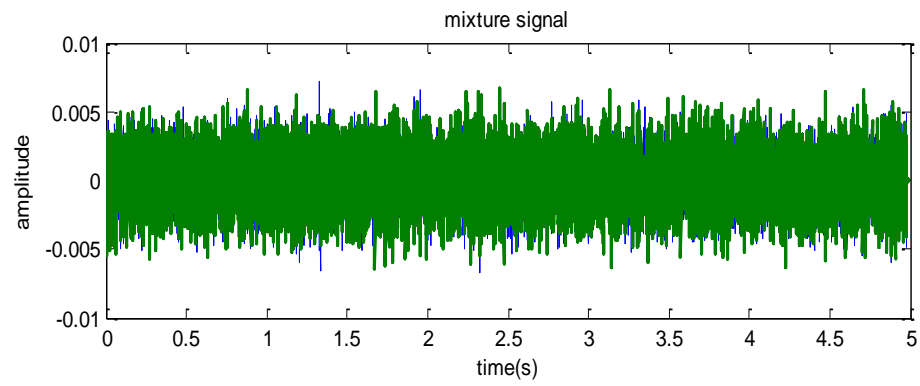

Figure 1. Mixture recording with two microphones in time domain

Profile matrix I, $\mathrm{J}$ and $\mathrm{K}$ will be in iterations until a minimum fitting function to obtain the estimates of the model that corresponds to the mixing process using a multi-way array decomposition. Permutations will be applied and adapted to $\mathrm{n}$ sources at all frequencies. In the clustering process, permutation will be applied based on envelop profiles. Correlation will be used to measure the degree of correspondence between the center point of cluster or centroid and envelop signal. In order to reduce the crosstalk that may occur, a demixing matrix will be re-estimated to crosstalk at each frequency and each sub-block is reduced. Furthermore, the signal in time-frequency domain will be transformed back to the time domain by performing inverse of short-time Fourier transform.

In order to obtain performance of proposed method in the time and frequency domain, we evaluated the estimated sources using mean square error (MSE) in the time domain and log spectral distance (LSD) in the frequency domain. LSD is the square of the difference between the spectral envelop logarithm of the original signal $b(n, f)$ and the estimated signal $\hat{b}(\mathrm{n}, \mathrm{f})[14]$. The estimated signals will be closer to the original source signals if LSD and MSE is getting smaller. In order to determine the location and diagnostic of machinery fault, we can use an instantaneous frequency approach [12].

$$
\begin{aligned}
& \text { MSE }=\frac{1}{\mathrm{~L}} \sum_{\mathrm{i}-1}^{\mathrm{L}}(\mathrm{b}(\mathrm{t})-\hat{b}(t))^{2} \\
& D_{L S}=\sqrt{\frac{1}{L} \sum_{n}\left(\log 10 \frac{b(n, f)}{\hat{b}(n, f)}\right)^{2}}
\end{aligned}
$$

where $D_{L S}$ is $\log$ spectal distance and $\mathrm{L}$ is number of samples

\subsection{Experiment}

In this study, we conducted an experiment in a real plant. The time interval of each signal recording is 5 seconds. Based on the measurements of the sound pressure level, reverberation time is $726 \mathrm{~ms}$. The length of fast Fourier transform is 1024 . The recording process is divided into two phases, namely the baseline and mixture recording. In baseline recording, we have a combination of any source with one sensor, where the sensor is placed $5 \mathrm{~cm}$ from the source. In mixture recording, we use a combination of four sources with 2 sensors. Sensors are placed $90 \mathrm{~cm}$ from the sources. Sources used in this study came from drive end-motor $B_{1}$, nondrive end-motor $B_{2}$, drive end pump $B_{3}$, and nondrive end pump $B_{4}$, as shown in Figure 2. Each part of the rotating machine has different characteristics depending on the condition of the machine.

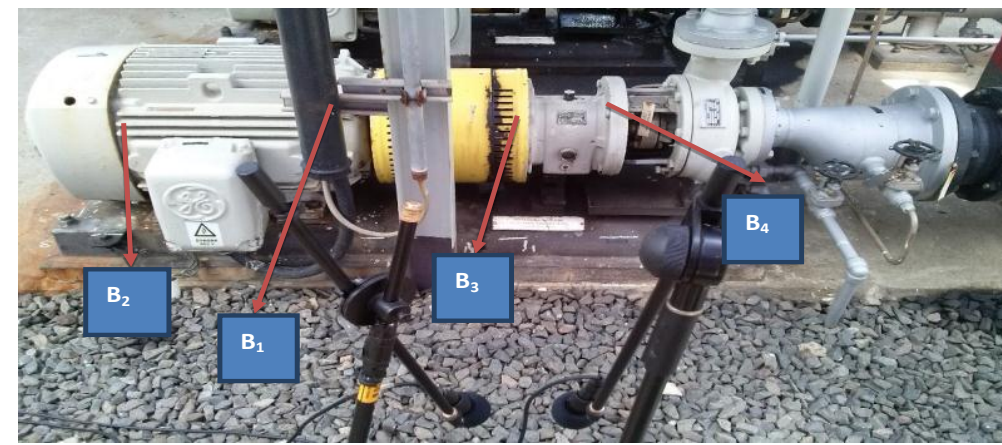

Figure 2. Recording process of mixtures in real plant using two microphones 


\section{RESULT AND DISCUSSION}

Mixed signals coming from four sources are separated by multi-way array decomposition method. The mixing matrix will be obtained after the function fitting on the multi-way array decomposition has reached the minimum value.

Based on Table 1, the smallest MSE value is 0.0707 on estimated signal $\hat{B}_{4}$ and the largest MSE is 0.1207 on the estimated signal $\hat{B}_{2}$. Based on LSD criterion in the frequency domain, the closest spectrum of estimated signal to the spectrum of the signal source is 1.914 on the estimated signal $\hat{B}_{4}$. The small value of the MSE and LSD means close to the baseline signal and vice versa in time and frequency domain. In this study, proposed method used will also be validated by the vibration signal in the frequency domain as described previously.

Vibration data is collected at the same time when recording an acoustic signal. According to the Figure 3 and Figure 4, there is a difference spectral of each machine both on an acoustic signal and the vibration signal.

Table 1. MSE and LSD between estimated signals and the baseline signal for each machine with two microphones

\begin{tabular}{ccc}
\hline Source Signal & MSE & LSD \\
\hline $\mathrm{B}_{1}$ & 0.0994 & 2.593 \\
$\mathrm{~B}_{2}$ & 0.1207 & 2.120 \\
$\mathrm{~B}_{3}$ & 0.0714 & 2.048 \\
$\mathrm{~B}_{4}$ & 0.0707 & 1.914 \\
\hline
\end{tabular}

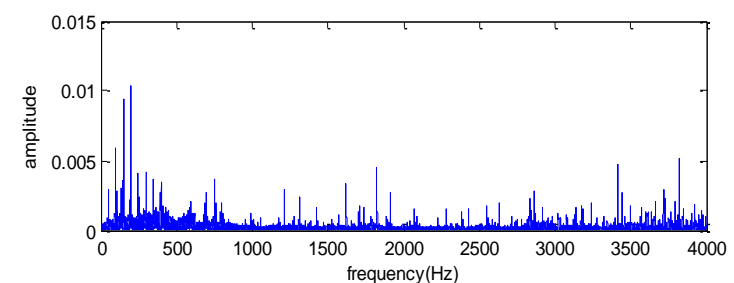

Estimated signal of $\mathrm{B}_{1}$

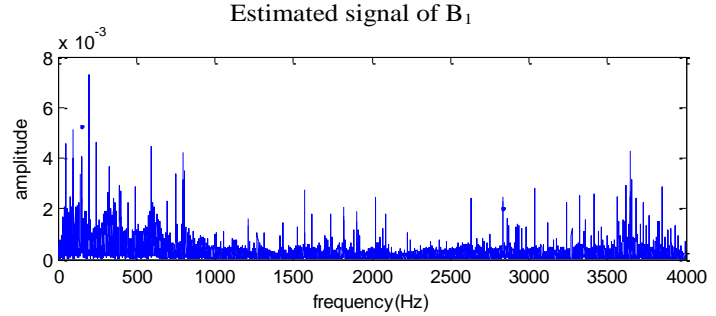

Estimated signal of $\mathrm{B}_{2}$

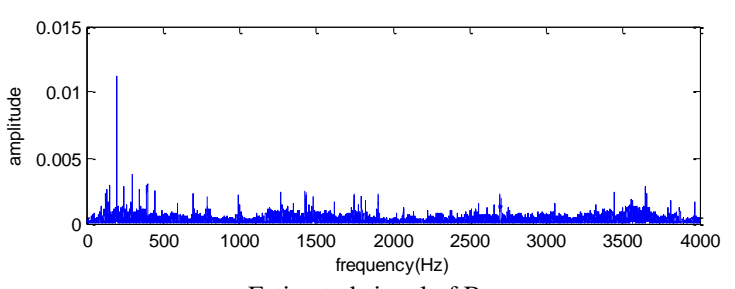

Estimated signal of $\mathrm{B}_{3}$

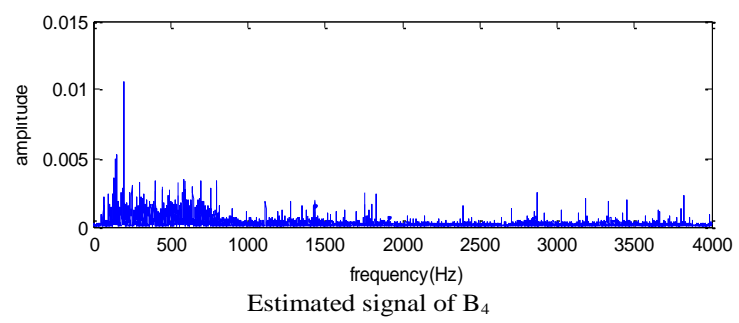

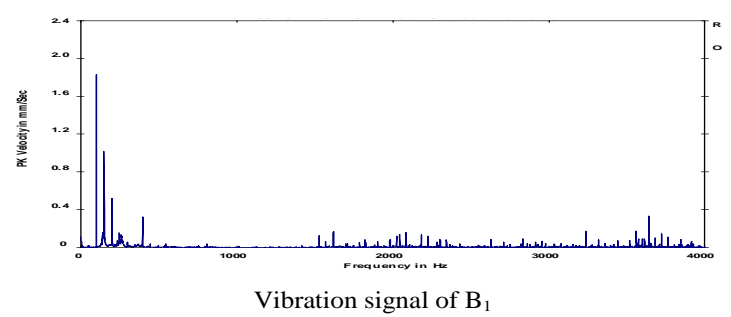
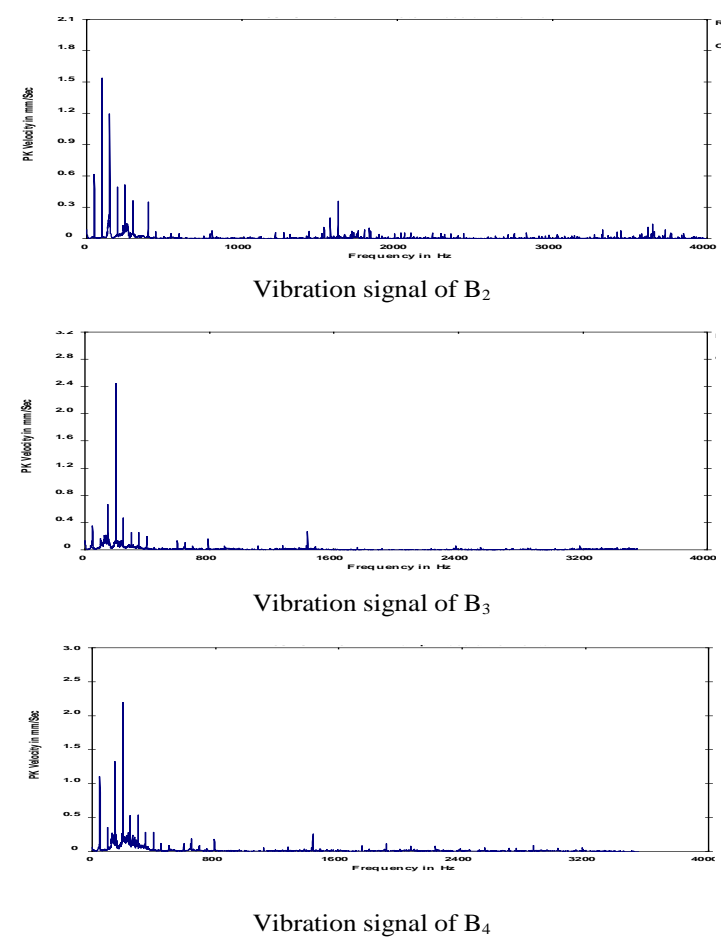

Figure 3. Comparison of spectrum between estimated and vibration signals 
The location of instantaneous frequency is also one of the important parameters in fault diagnosis. The instantaneous frequency comparison of the estimation signal and vibration signal can be seen in Table 2 . In the vibration analysis, the instantaneous frequency location will be compared with the fundamental frequency so that the order parameter can be obtained. Based on the order such as $1 \mathrm{x}, 2 \mathrm{x}, 3 \mathrm{x}$ and so on as well as the spectrum pattern of the machine, then the location of machinery fault can be identified.

The frequency domain of the experimental result which is characteristic of the vibration derived from the physical properties of the machinery components due to the imbalance forces of the motor components. Based on the vibration spectrum analyzer, the motor has a local defect on the outer race. It is indicated with ball pass frequency outer (BPFO) pattern, the detail shown in Figure 4(a). It shows that there is friction between the ball bearing movement with outer race causing the transfer of force from the crankshaft to the motor body. It is clearly shown on the spectrum of nondrive end-motor $\mathrm{B}_{2}$ marked by the harmonic peak along the frequency bins. In the vibration signal, the order of source signal $\hat{B}_{2}$ is located at $4.086 \mathrm{x}$ fundamental frequency of the machine. It has a harmonic frequency spectrum with $0.105 \mathrm{~mm} / \mathrm{sec}$ amplitude which is marked by the dashed lines in Figure 4(a). The estimated spectrum of source signal $\hat{B}_{2}$ as seen in Figure 4 is indicated as same as fault condition in vibration spectrum. The order of $\mathrm{B}_{2}$ can be obtained from the instantaneous frequency $(202.55 \mathrm{~Hz})$ divided by fundamental frequency of machine $(49.57 \mathrm{~Hz})$. The fundamental frequency can be seen in Figure 4 obtained from the vibration spectrum of the machine. The indication of misalignment can be shown in Figure 4(b) and Figure 5(b) that drive end-pump $\mathrm{B}_{3}$ has a high peak in $198 \mathrm{~Hz}$ or $4 \mathrm{x}$ fundamental frequency. The spectrum of an acoustic signal has background noise that is considerably higher than the spectrum of a vibration signal. However, it still has suitability with the spectrum of a vibration signal for fault diagnosis.

Table 2. Comparison of instantaneous frequency between estimated and vibration signals

\begin{tabular}{ccc}
\hline Source Signal & \multicolumn{2}{c}{ Instantaneous frequency $(\mathrm{Hz})$} \\
& Estimated signal & Vibration signal \\
\hline $\mathrm{B}_{1}$ & 198.21 & 203.01 \\
$\mathrm{~B}_{2}$ & 247.77 & 202.55 \\
$\mathrm{~B}_{3}$ & 198.65 & 198.62 \\
$\mathrm{~B}_{4}$ & 151.42 & 198.26 \\
\hline
\end{tabular}

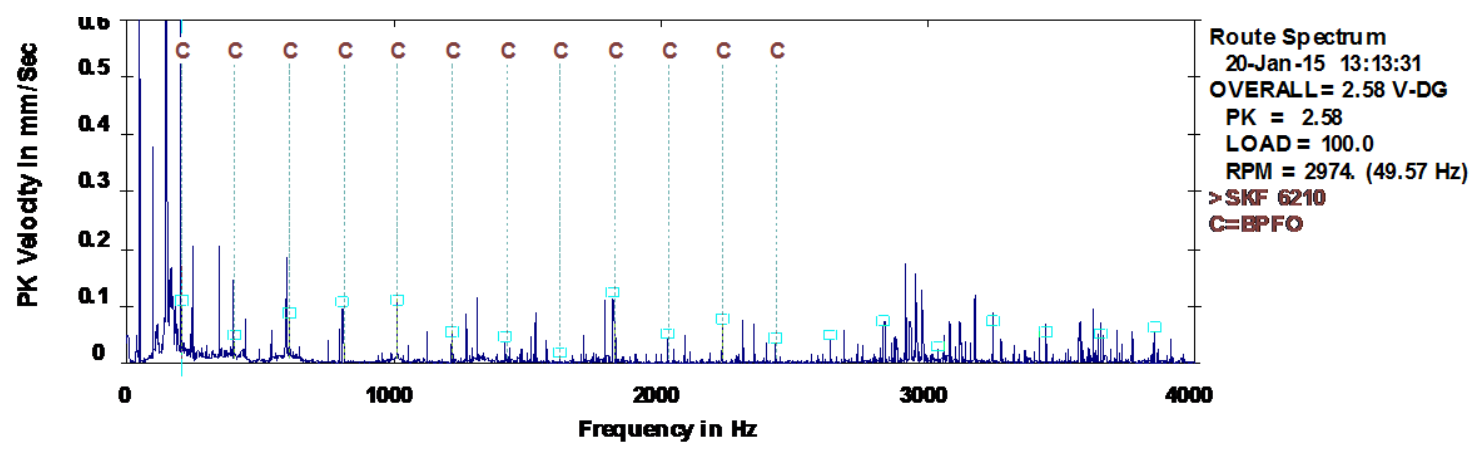

(a)

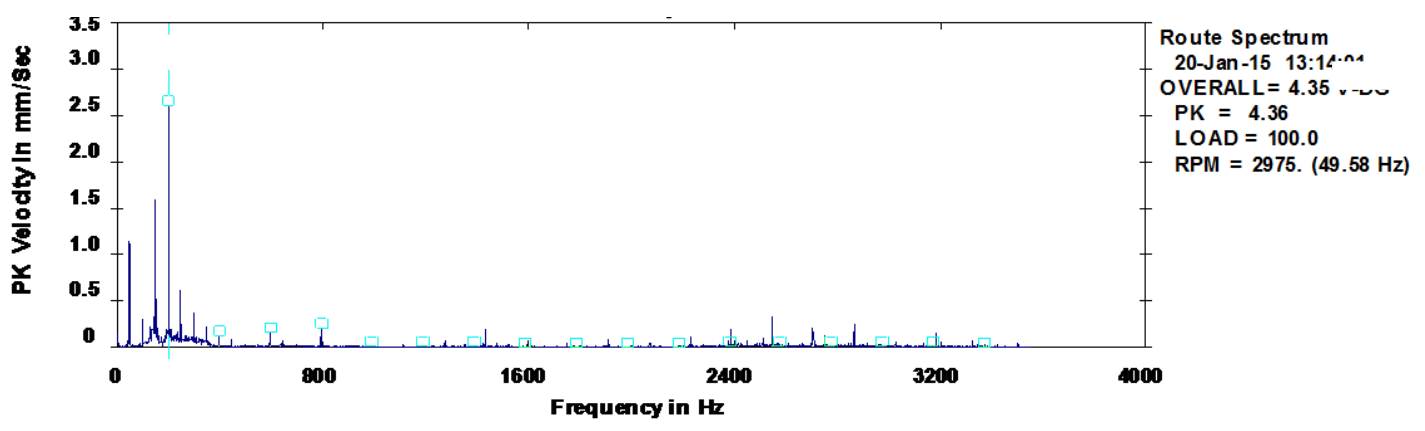

(b)

Figure 4. Diagnostic of outer race bearing fault and misalignment from vibration spectrum analyzer (a) $B_{2}$ and (b) $\mathrm{B}_{3}$ 


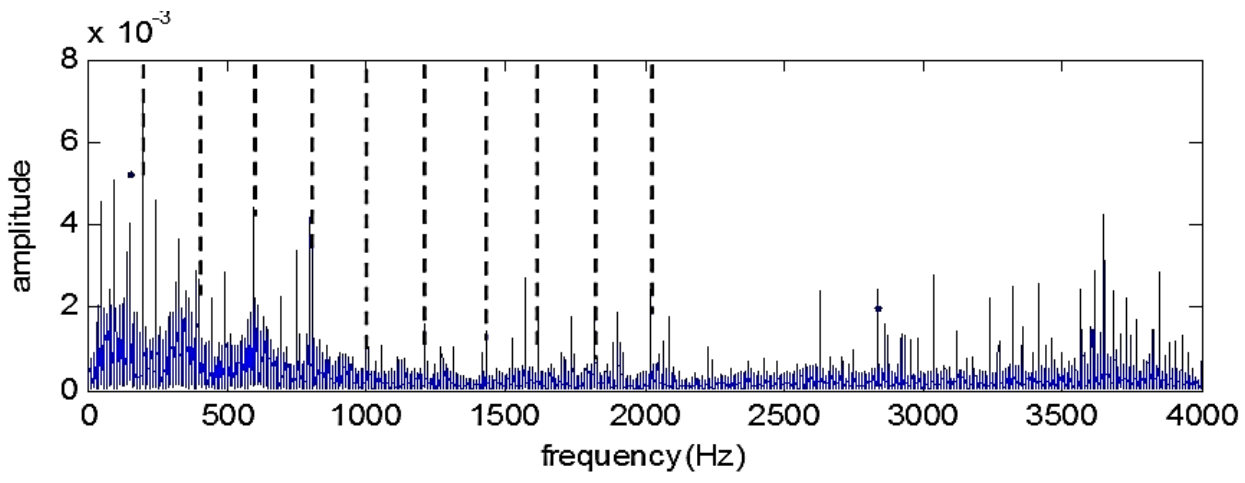

(a)

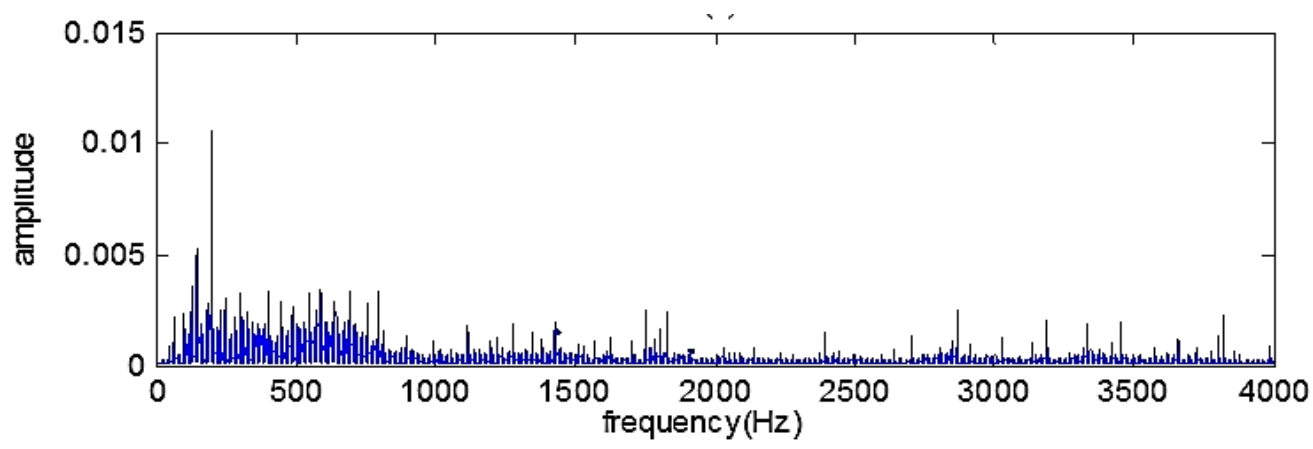

(b)

Figure 5. Diagnostic of outer race bearing fault and misalignment from estimated acoustic spectrum (a) $\hat{B}_{2}$ and (b) $\hat{B}_{3}$

\section{CONCLUSION}

This paper presents diagnostics of machinery fault based on acoustic emission. Sources used in this study came from drive end-motor $B_{1}$, nondrive end-motor $B_{2}$, drive end pump $B_{3}$, and nondrive end pump $B_{4}$ Mixed signals coming from four sources are separated by multi-way array decomposition approach. The approach can solve the complexity of approximate joint diagonalization process in the underdetermined case. In this study, we conducted an experiment in a real plant with two microphones. Based on the results obtained, the types of machinery fault such as misalignment and outer bearing fault can be detected by acoustic signal estimation. The performance of proposed method will be evaluated using MSE and LSD. Based on the result, the smallest MSE value is 0.0707 on estimated signal $\hat{B}_{4}$ and the largest MSE is 0.1207 on the estimated signal $\hat{B}_{2}$. Based on LSD criterion in the frequency domain, the closest spectrum of estimated signal to the spectrum of the signal source is 1.914 on the estimated signal $\hat{B}_{4}$. The small value of the MSE and LSD means close to the baseline signal and vice versa in time and frequency domain. Based on the comparison of acoustic signals and vibration signals, the diagnostic of outer race defect and misalignment from estimated spectrum of $\widehat{B}_{2}$ and $\hat{B}_{3}$ is indicated as same as vibration spectrum of $\mathrm{B}_{2}$ and $\mathrm{B}_{3}$.

\section{ACKNOWLEDGEMENTS}

The authors would like to thank Lembaga Penelitian dan Pengabdian Masyarakat (Institute of Research and Society Development) - Universitas Internasional Semen Indonesia who has provided financial support for this publication.

\section{REFERENCES}

[1] Balerston, H. L, "The detection of incipient failure in bearings," Materials Evaluation, Vol 27, 121-128, 1969.

[2] Lebaroud, Abdesselam, and Guy Clerc, "Diagnosis of induction motor faults using instantaneous frequency signature analysis," 18th International Conference on Electrical Machine IEEE, 2008. 
[3] Ayaz, Emine, et al, "Fault detection based on continuous wavelet transform and sensor fusion in electric motors," COMPEL-The international journal for computation and mathematics in electrical and electronic engineering 28.2 (2009): 454-470.

[4] Wang, Changqing, et al, "Fault diagnosis based on pulse coupled neural network and probability neural network," Expert Systems with Applications 38.11 (2011): 14307-14313.

[5] Comon, Pierre, and Christian Jutten, eds, "Handbook of Blind Source Separation: Independent component analysis and applications," Academic press, 2010.

[6] M. Zibulevsky, "Relative Newton method for quasi-ML blind source separation," In Proc. 4th Intern. Symp. on Independent Component Analysis and Blind Signal Separation (ICA2003), pages 897\{902, Nara, Japan, 2003.

[7] A. Belouchrani, K. Abed-Meraim, M. G. Amin and A. M. Zoubir, "Joint anti-diagonalization for blind source separation," Acoustics, Speech, and Signal Processing, 2001. Proceedings. (ICASSP '01). 2001 IEEE International Conference on, Salt Lake City, UT, 2001, pp. 2789-2792 vol.5.

[8] Cui, Lingli, et al, "Diagnosis of Roller Bearings Compound Fault Using Underdetermined Blind Source Separation Algorithm Based on Null-Space Pursuit," Shock and Vibration, 2015.

[9] A. A. Vinaya and D. Arifianto, "Underdetermined blind source separation based condition monitoring," 2015 International Conference on Science in Information Technology (ICSITech), Yogyakarta, 2015, pp. 47-52.

[10] Müller, Thorsten, et al, "Fault Detection with Qualitative Models reduced by Multi-way array Decomposition methods," IFAC-PapersOnLine 48.21 (2015): 416-421.

[11] Nion, Dimitri. (2010), "Batch and Adaptive PARAFAC-Based Blind Separation of Convolutive Speech Mixtures," IEEE Transactions On Audio, Speech, And Language Processing, Vol. 18, No. 6.

[12] Condition monitoring Team.Vibration training course book, Mobius Institute, 2005.

[13] Felten, Dave, "Understanding bearing vibration frequencies," Electrical Apparatus Service Association (EASA), St. Louis, MO, 2003.

[14] Freudenberger, J., Stenzel, S., \& Venditti, B. (2010), "Microphone diversity combining for in-car applications," EURASIP Journal on Advances in Signal Processing, vol 5.

\section{BIOGRAPHIES OF AUTHORS}
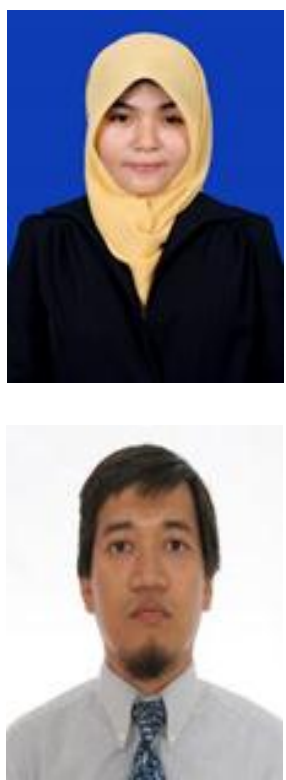

Anindita Adikaputri Vinaya received a master's degree in engineering physics from Institut Teknologi Sepuluh Nopember, Surabaya in 2015. Her research focus on acoustic separation\&localization, instrumentation and risk management. She is presently working as lecturer in Universitas Internasional Semen Indonesia.

Dhany Arifianto completed a bachelor's degree in engineering physics from Institut Teknolog Sepuluh Nopember, Surabaya. He received M.Eng degree in 2002 and Dr.Eng degree in 2005 from Tokyo Institute of Technology. His research interests related to digital signal processing and convex optimization. He has several journal and conference publications in national and international level. He is an active member of Hitachi and IEEE. He has also served as keynote speaker in several international conferences. 